Article

\title{
An Inducible Cre-lox System to Analyze the Role of LLO in Listeria monocytogenes Pathogenesis
}

\author{
Brittney N. Nguyen ${ }^{1}$ (D) and Daniel A. Portnoy ${ }^{2,3, *}$ \\ 1 Graduate Group in Microbiology, University of California, Berkeley, CA 94720, USA; \\ brittneynguyen@berkeley.edu \\ 2 Department of Molecular and Cell Biology, University of California, Berkeley, CA 94720, USA \\ 3 Department of Plant and Microbial Biology, University of California, Berkeley, CA 94720, USA \\ * Correspondence: portnoy@berkeley.edu
}

Received: 9 November 2019; Accepted: 31 December 2019; Published: 7 January 2020

\begin{abstract}
Listeriolysin O (LLO) is a pore-forming cytolysin that allows Listeria monocytogenes to escape from phagocytic vacuoles and enter the host cell cytosol. LLO is expressed continuously during infection, but it has been a challenge to evaluate the importance of LLO secreted in the host cell cytosol because deletion of the gene encoding LLO (hly) prevents localization of L. monocytogenes to the cytosol. Here, we describe a L. monocytogenes strain $(h l y l)$ in which $h l y$ is flanked by loxP sites and Cre recombinase is under the transcriptional control of the L. monocytogenes act $A$ promoter, which is highly induced in the host cell cytosol. In less than $2 \mathrm{~h}$ after infection of bone marrow-derived macrophages (BMMs), bacteria were $100 \%$ non-hemolytic. $h l y$ fl grew intracellularly to levels 10 -fold greater than wildtype L. monocytogenes and was less cytotoxic. In an intravenous mouse model, 90\% of bacteria were non-hemolytic within three hours in the spleen and eight hours in the liver. The loss of LLO led to a 2-log virulence defect in the spleen and a 4-log virulence defect in the liver compared to WT L. monocytogenes. Thus, the production of LLO in the cytosol has significant impact on the pathogenicity of L. monocytogenes.
\end{abstract}

Keywords: pathogenesis; cytotoxicity; pore-forming toxin; cholesterol-dependent cytolysin; vaccine

Key Contribution: The construction and characterization of a L. monocytogenes strain that deletes hly in the host cell cytosol to study the effects of LLO secreted in the cytosol.

\section{Introduction}

The field of microbial pathogenesis and the study of virulence factors has been guided for decades by Molecular Koch's Postulates, which stipulate that inactivation of a gene encoding a suspected virulence factor should lead to measurable loss of virulence, and replacement of the gene should restore pathogenicity [1]. Although targeted gene deletions are invaluable in determining the function of genes and pathways, there remain circumstances in which it is not possible to generate viable deletion mutants, or deletion of a gene encoding multiple functions precludes analysis of later functions. The latter is the case for the gene encoding Listeriolysin $\mathrm{O}(\mathrm{hly})$ of Listeria monocytogenes.

L. monocytogenes is a Gram-positive facultative intracellular pathogen that specifically replicates in the cytosol of host cells. In order to reach the host cell cytosol, L. monocytogenes must first escape from the phagocytic entry vacuole, which requires the secreted pore-forming cytolysin Listeriolysin $\mathrm{O}$ (LLO) [2,3]. In the cytosol, L. monocytogenes replicates and produces an actin nucleation factor (ActA) to move intracellularly and form protrusions that are engulfed by neighboring cells and resolved into double-membraned vacuoles. Again, LLO is required for escape from these secondary vacuoles $[4,5]$. 
LLO belongs to a large family of cholesterol-dependent cytolysins (CDCs) which also includes: Perfringolysin of Clostridium perfringens, Streptolysin O of Streptococcus pyogenes, and Pneumolysin of Streptococcus pneumoniae [6]. Importantly, LLO is the only CDC produced by an intracellular pathogen and its roles in pathogenesis are distinct and unique compared to related CDCs [7].

Though the only clearly established role of LLO during infection is inside of L. monocytogenes-containing vacuoles, LLO is continuously secreted inside host cells. The continuous secretion of LLO has the potential to form pores in the host cell membrane and cause cell death. Mechanisms have been identified that suppress LLO activity within the host cytosol, lessening its potential toxicity, including: reduced activity at the neutral $\mathrm{pH}$ of the cytosol compared to the acidic $\mathrm{pH}$ of the vacuole, degradation by the proteasome, and translational repression by the $5^{\prime}$ coding sequence [8-11]. Additionally, LLO co-opts host endocytosis machinery for removal of LLO from the plasma membrane [12]. Mutations that abolish any of these control mechanisms increase L. monocytogenes cytotoxicity [13]. However, other than cell death, these studies do not address the potential activities that LLO has when secreted in the cytosol.

Despite the numerous mechanisms that suppress LLO activity in the cytosol, it is still not clear whether LLO is suppressed to the point where it has no activity. Unfortunately, whether LLO secreted in the cytosol has any effects has been difficult to address because of the early requirement for LLO, although strategies have been developed to examine this question. To show that LLO was required for escape from primary single-membrane and secondary double-membrane vacuoles, Gedde et al. noncovalently coupled purified LLO to $\Delta h l y$ L. monocytogenes and observed that the bacteria were able to escape from the primary vacuole, but because of their inability to produce more LLO they became trapped in secondary vacuoles [4]. Similarly, Dancz et al. demonstrated that L. monocytogenes expressing IPTG-inducible LLO remain trapped in vacuoles until addition of IPTG [5]. Czuczman et al. studied L. monocytogenes in HeLa cells, in which LLO is not required for escape from the vacuole, and concluded that LLO causes localized plasma membrane damage that allows L. monocytogenes to hijack the cell efferocytosis machinery for cell-to-cell spread [14]. Others have observed that LLO disrupts SUMOylation, modifies histones, and causes mitochondrial fragmentation during infection by simply adding purified LLO exogenously to cells [15-19]. These studies have all tried to circumvent the requirement for LLO in the vacuole, but the diversity in the techniques used and their limitations makes it a challenge to integrate the conclusions into a complete picture. Additionally, these studies have not addressed the effects of these activities in mice.

We have developed a tool for studying LLO that does not circumvent the requirement for LLO in vacuolar escape. We engineered a strain of L. monocytogenes that initially produces LLO, allowing it to escape from the vacuole. After escape from the vacuole hly is excised by Cre-lox-mediated DNA recombination and the strain becomes a $\Delta h l y$ mutant. Here, we report rapid excision of hly in bone marrow-derived macrophages (BMMs) and in a mouse model. In BMMs, LLO secreted in the cytosol contributes to significant amounts of cytotoxicity. $h l y^{\mathrm{fl}}$ grew to a level 10-fold higher than wildtype (WT) L. monocytogenes in BMMs, likely due to a reduction in cell death.

\section{Results}

\subsection{Use of hlyfl in Cultured Cells}

\subsubsection{Cre-lox Allows for Rapid Excision of hly during Infection of Macrophages}

To study the role of LLO secreted in the cytosol during infection, we engineered a strain of L. monocytogenes, called $h l y^{\mathrm{fl}}$, to excise the gene encoding LLO, hly, following escape of L. monocytogenes from the phagocytic vacuole. Specifically, loxP sites were inserted into the L. monocytogenes chromosome to flank $h l y$ and an adjacent gene, tet $L$, which provides tetracycline resistance. Cre recombinase, which mediates DNA recombination between loxP sites, was inserted into the chromosome using the pPL2 integrative vector, and expressed under the control of the L. monocytogenes act $A$ promoter, which is 
relatively inactive prior to vacuolar escape of L. monocytogenes and becomes highly expressed in the cytosol (Figure 1A). Thus, this strain is able to produce LLO initially to facilitate escape from the phagocytic vacuole but, once in the cytosol, hly is excised and LLO production ceases. To determine the efficiency of the system, BMMs were infected with $h l y^{\mathrm{fl}}$ L. monocytogenes and bacteria from the infected cells were recovered at different time points and plated on blood-agar media. Secreted LLO causes rapid $\beta$-hemolysis and $L$. monocytogenes colonies that secrete LLO can be easily identified (Figure 1B). Prior to infection, $h l y^{\mathrm{fl}}$ L. monocytogenes were grown in broth containing tetracycline to select against low-level excision of hly and tetL. By $30 \mathrm{~min}$ post-infection almost $90 \%$ of recovered colony-forming units (CFU) were non-hemolytic (Figure 1C). By 60 min post-infection, $98 \%$ of recovered CFU were non-hemolytic and by $90 \mathrm{~min}$ post-infection all colonies were non-hemolytic. Therefore, the excision of $h l y$ is rapid and complete during infection of BMMs.

A

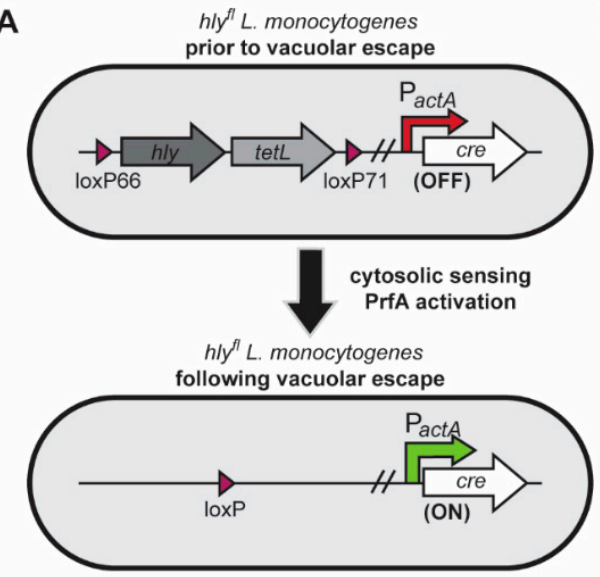

C

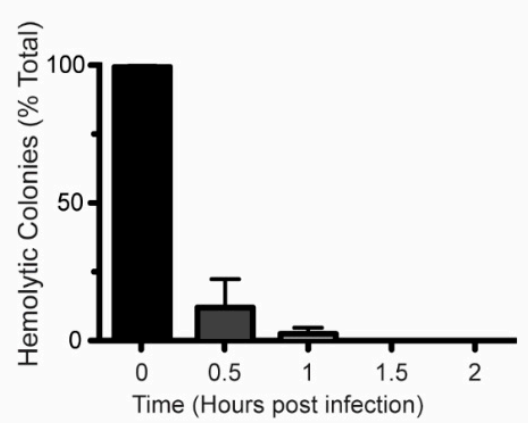

B

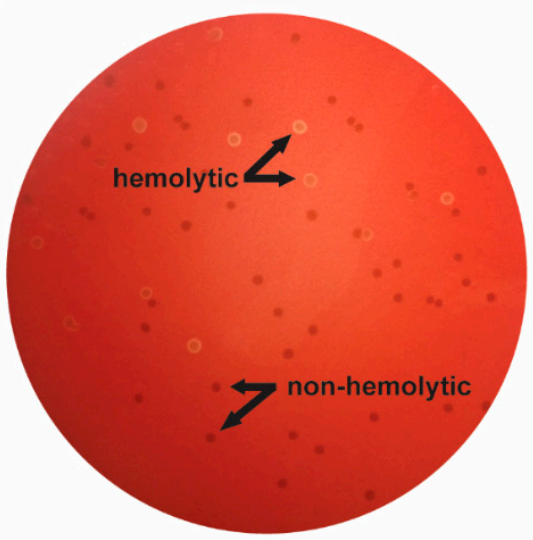

Figure 1. $h l y$ is excised in the $h l y^{\mathrm{fl}}$ strain in bone marrow-derived macrophages (BMMs) through DNA recombination. (A) Schematic of $h l y \mathrm{fl}$ L. monocytogenes strain. The hly and tet $L$ (tetracycline resistance) genes are flanked by loxP sites. Cre recombinase is controlled by the act $A$ promoter. Recombination between loxP sites leads to the excision of the DNA encoding hly and tetL (1); (1): Reprinted from Cell Host \& Microbe, Vol 23/Issue 6, Chen Chen, Brittney N. Nguyen, Gabriel Mitchell, Shally R. Margolis, Darren Ma, Daniel A. Portnoy, The Listeriolysin O PEST-like Sequence Co-opts AP-2-Mediated Endocytosis to Prevent Plasma Membrane Damage during Listeria Infection, 786-795., Copyright (2018), with permission from Elsevier. (B) Hemolytic and non-hemolytic colonies of $h l y^{\mathrm{fl}}$. (C) To quantify the excision of $h l y$, BMMs were infected with $h l y \mathrm{fl}$ and both hemolytic and non-hemolytic colonies were enumerated by plating bacteria on blood-agar media at different timepoints. Mean and SD of data pooled from three independent experiments are shown.

\subsubsection{LLO Secreted in the Cytosol Affects Intracellular Growth and Contributes to Cytotoxicity}

To determine whether secretion of LLO by L. monocytogenes in the cytosol affects the growth of the bacteria in cells, intracellular growth was evaluated in BMMs. During the first five hours of infection, $h l y^{\mathrm{fl}}$ L. monocytogenes grew identically to WT L. monocytogenes and $\triangle$ act $A$, which is defective in actin-based motility and therefore defective in cell-to-cell spread (Figure 2A). However, after five 
hours of infection, the growth of the strains diverged. Between five and twenty-four hours of infection, the number of WT L. monocytogenes plateaued and then declined, but the number of recovered $h l y^{\mathrm{fl}}$ L. monocytogenes increased to 10-fold more than the maximum of WT and remained elevated, suggesting that secretion of LLO in the cytosol negatively impacts growth of WT.
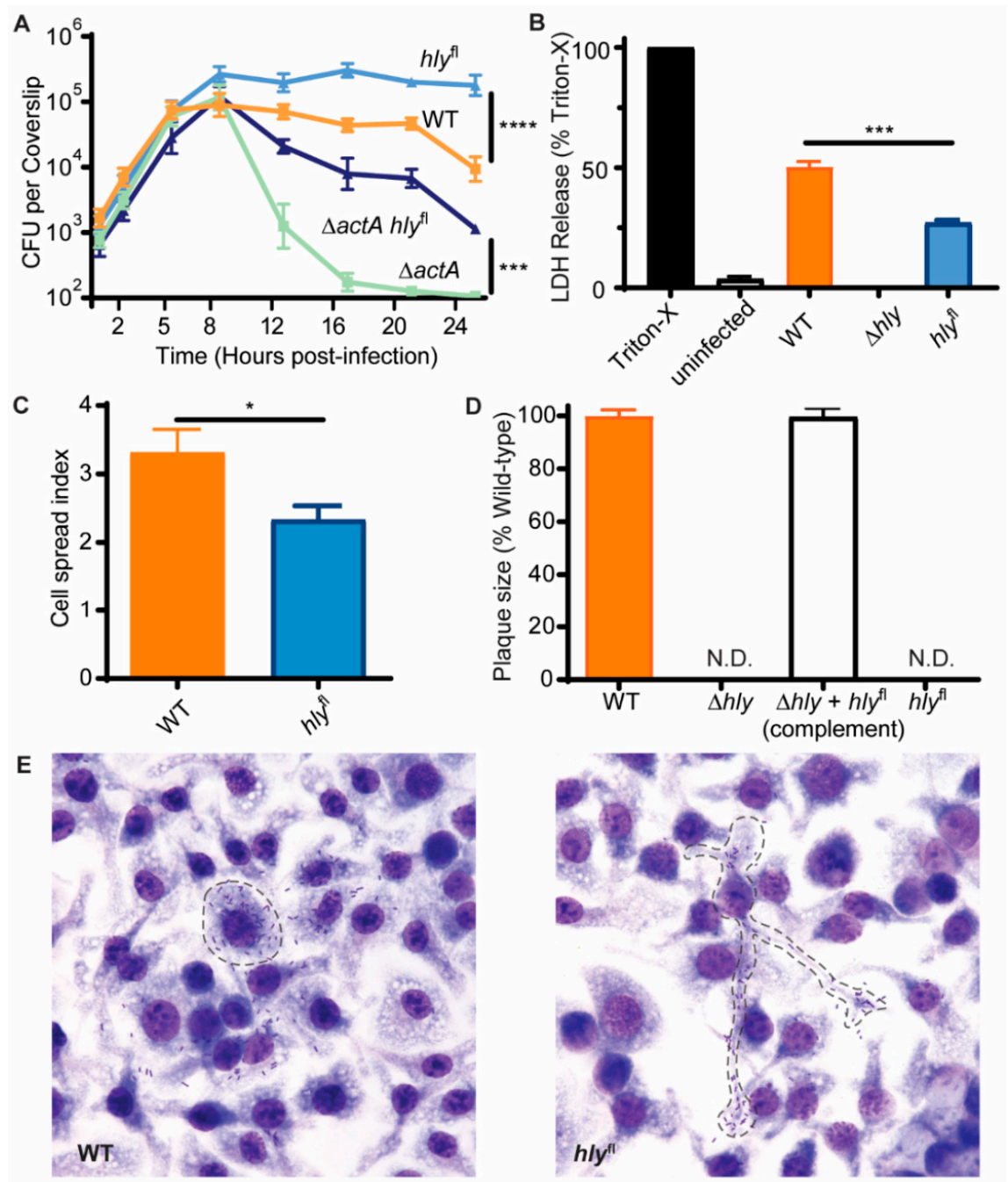

Figure 2. $h l y \mathrm{fl}^{\mathrm{fl}}$ grew intracellularly and had reduced cytotoxicity but had a defect in cell-to-cell spread. (A) BMMs were infected at an MOI of 0.17 with L. monocytogenes and intracellular CFU were enumerated at different times. Three biological replicates were measured in each experiment, and experimental results were combined. For $\Delta a c t A$ and $\triangle a c t A h l y^{\mathrm{fl}} n=6$. For WT and $h l y^{\mathrm{fl}} n=9$. Mean and SEM are shown. Results were log transformed and means were compared using Holm-Sidak's multiple comparisons test. (B) BMMs were infected with L. monocytogenes at an MOI of 4 for $24 \mathrm{~h}$. Lactate dehydrogenase $(\mathrm{LDH})$ release was measured and values were normalized according to lysis with $1 \%$ Triton-X 100 representing 100\% LDH release and the condition with the smallest mean value representing $0 \% \mathrm{LDH}$ release. The average of three technical replicates per experiment, from three independent experiments, are presented. Means were compared using an unpaired $t$-test. (C) BMMs were infected with $L$. monocytogenes and cell-to-cell spread was quantified $5 \mathrm{~h}$ post-infection. WT $n=67, h l y^{\mathrm{fl}} n=60$. Means were compared using an unpaired $t$-test. (D) L2 cells were infected with L. monocytogenes at an MOI of 0.1. Plaque size was measured after 3 days and values were normalized to WT plaque size. Mean and SEM for data pooled from three independent experiments is shown. WT $n=19, \Delta h l y+h l y^{\mathrm{fl}} n=14$. N.D. indicates no plaques were detectable. (E) Representative images of the BMMs infected with L. monocytogenes for cell-to-cell spread analysis in (C). Dashed lines are used to indicate the initially-infected cells. 
WT L. monocytogenes has the ability to spread to and replicate in neighboring cells. If $h l y^{\mathrm{fl}}$ has a defect in escape from secondary vacuoles that would limit its ability to replicate in neighboring cells, the difference in growth between WT and $h l y^{\mathrm{fl}}$ may reflect both the effects of LLO secreted in the cytosol and growth following cell-to-cell spread. To analyze the effects of LLO in the cytosol without complication by cell-to-cell spread, we compared growth of $h l y^{\mathrm{fl}}$ in a $\triangle a c t A$ background $\left(\triangle a c t A h l y^{\mathrm{fl}}\right)$ to $\triangle a c t A$, which is defective in actin-based motility and therefore defective in cell-to-cell spread. During the first eight hours of infection, $\triangle a c t A$ and $\triangle a c t A h l y^{\mathrm{fl}}$ grew similarly to WT. However, between eight and twenty-four hours, the number of $\triangle a c t A$ bacteria decreased dramatically, whereas $\triangle a c t A h l y \mathrm{fl}^{\mathrm{fl}}$ decreased much less-having as much as 100-fold more bacteria than $\triangle a c t A$. The rapid loss of $\triangle a c t A$ CFU, which is due to the influx of gentamicin [20], was partially rescued by deletion of hly in the cytosol, indicating that the decline of $\triangle a c t A$ in cells is partly LLO dependent.

The ability of LLO to form pores in cholesterol-containing cell membranes is well documented. We hypothesized that the growth of WT L. monocytogenes could be restricted by LLO-induced cytotoxicity because LLO has the potential to bind to the cell membrane and cause cell death. To quantify the amount of cell death caused by L. monocytogenes infection, cytotoxicity was measured by lactate dehydrogenase (LDH) release assay (Figure $2 \mathrm{~B}$ ). After a $24 \mathrm{~h}$ infection of BMMs, $43 \%$ of cells were killed by WT L. monocytogenes infection. Only 22\% of cells were killed by hly fl L. monocytogenes infection, indicating that LLO secreted in the cytosol contributes significantly to cytotoxicity during infection.

Previously, a requirement for LLO in escaping double-membraned phagocytic vacuoles after cell-to-cell spread was demonstrated using IPTG-inducible LLO [5]. Because hly fl should delete $h l y$ in the cytosol of the first cell it infects, we expected it to also be defective in escape from secondary vacuoles. To examine the fate of $h l y^{\mathrm{fl}}$, we performed cell spreading and plaque assays to measure cell-to-cell spread (Figure 2C-E). The number of cells in infectious foci were quantified for BMMs infected with WT and $h l y$ fl for $5 \mathrm{~h}$. On average, WT L. monocytogenes spread to two to three neighboring cells over a $5 \mathrm{~h}$ period, while $h l y{ }^{\mathrm{fl}}$ was only found in an average of two neighboring cells (Figure 2C). Furthermore, WT L. monocytogenes was often well distributed in cells of an infectious focus, indicating that bacterial replication had occurred in neighboring cells following cell-to-cell spread. In contrast, infectious foci of $h l y^{\mathrm{fl}}$ were often observed with one macrophage harboring the majority of bacteria and limited numbers of bacteria in the surrounding cells, suggesting that $h l y \mathrm{fl}$ spread to neighboring cells, but had a subsequent vacuolar escape defect (Figure 2E). To assess the impact on cell-to-cell spread resulting from hly deletion over a longer time period, L2 cells were infected with WT and $h l \mathrm{fl}^{\mathrm{fl}}$ L. monocytogenes. WT L. monocytogenes forms plaques in a monolayer of L2 cells by spreading from cell-to-cell. Both $h l y^{\mathrm{fl}}$ and $\Delta h l y$ L. monocytogenes were unable to form plaques, indicating that they cannot efficiently spread cell-to-cell. $\Delta$ hly that was complemented with $h l y$ fl but missing Cre recombinase, and therefore unable to excise hly, was restored to WT L. monocytogenes levels of plaque formation (Figure 2D). These results suggest that though LLO is not required for growth in cells, its continued production contributes to cell-to-cell spread.

\subsection{Use of hly fl in Mice}

\subsection{1. $h l y$ is Excised In Vivo and Its Excision Reduces Virulence}

To quantify the efficiency of the $h l y \mathrm{fl}$ Cre-lox system in vivo, C57BL/6J mice were infected intravenously with $10^{5} \mathrm{CFU}$ of $h l y^{\mathrm{fl}}$ L. monocytogenes (Figure 3). Hemolytic capacity of the inoculum was verified by plating on blood agar. At 1, 2, 3, 5, 8, and $24 \mathrm{~h}$ post-infection, bacteria were recovered from the spleen and liver and plated on blood agar and both hemolytic and non-hemolytic CFU were enumerated. In the spleen (Figure 3A), less than $20 \%$ of bacteria were hemolytic one hour post-infection. Hemolytic bacteria represented less than $2 \%$ of the population three hours post-infection and were nearly undetectable five hours post-infection. Then, $24 \mathrm{~h}$ post-infection, a small population of hemolytic bacteria were detected in the spleen (see discussion). In the liver (Figure 3B), excision of hly was slower than in the spleen. One hour post-infection, only 35\% of bacteria were non-hemolytic; eight 
hours post-infection $95 \%$ of bacteria were non-hemolytic and hemolytic colonies were undetectable by $24 \mathrm{~h}$ post-infection.

A

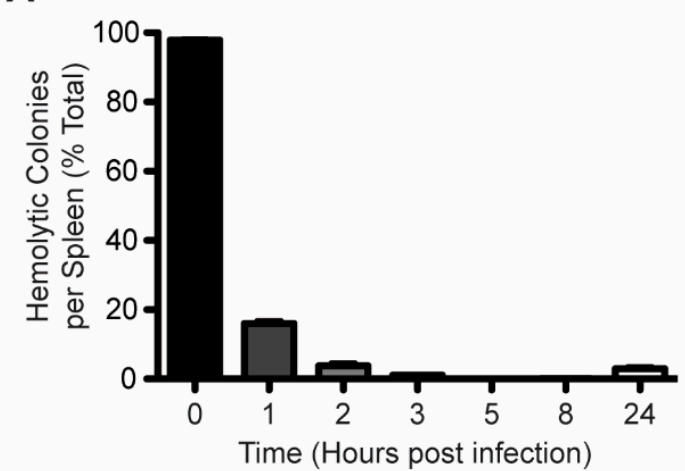

C

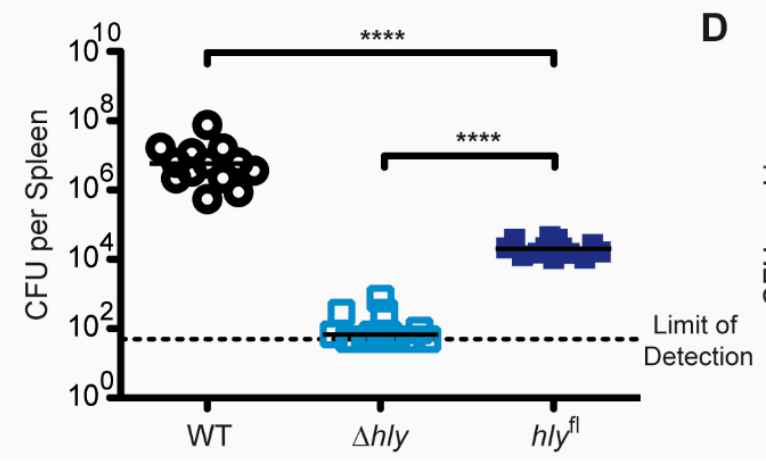

B
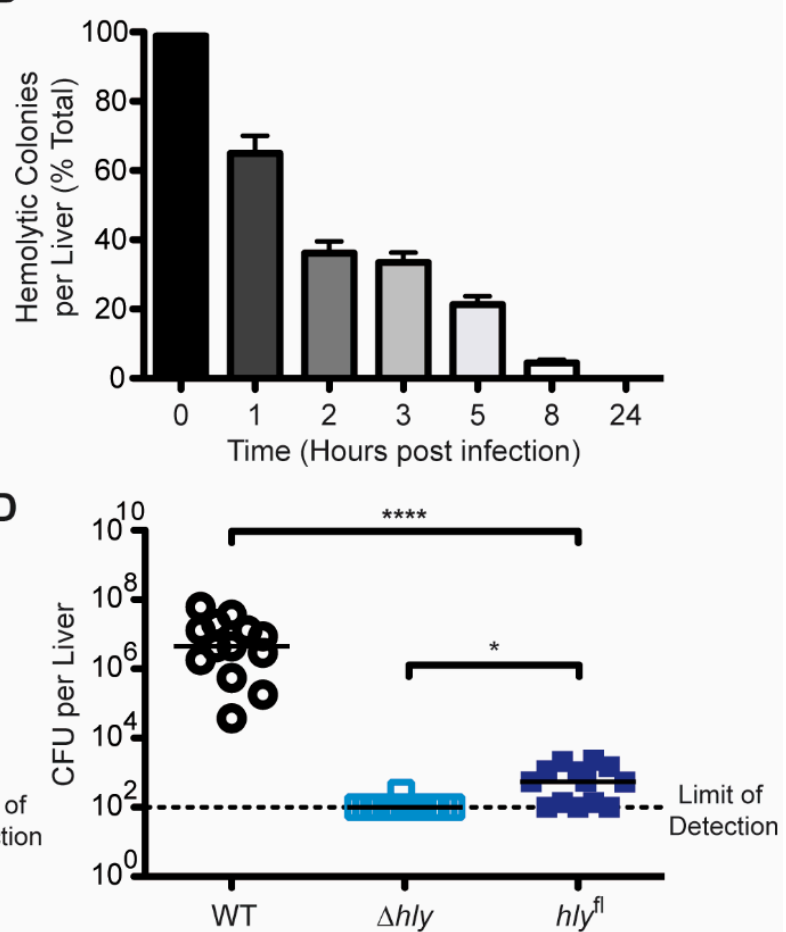

Figure 3. $h l y^{\mathrm{fl}}$ recombines in vivo and is attenuated in mice. (A,B) C57BL/6J mice were infected with $1 \times 10^{5} \mathrm{CFU}$ of $h l y^{\mathrm{fl}}$ L. monocytogenes. For each timepoint, hemolytic and non-hemolytic colonies recovered from the (A) spleen and (B) liver were enumerated. \% Total hemolytic colonies was calculated for each mouse. Mean and SEM are shown. Data are pooled from two independent experiments. For each timepoint, $n=6$. (C,D) CD-1 mice were infected with $1 \times 10^{5}$ CFU of L. monocytogenes. Then, $48 \mathrm{~h}$ post-infection, CFU from the (C) spleen and (D) liver were enumerated. Data are pooled from three independent experiments and medians are shown. $n=13$. Means were compared using Holm-Sidak's multiple comparisons test.

To determine the importance of LLO after escape of L. monocytogenes from the initial vacuole, we characterized $h l y^{\mathrm{fl}}$ L. monocytogenes using a mouse model of virulence. CD-1 mice were infected intravenously with $10^{5} \mathrm{CFU}$ of $L$. monocytogenes and $48 \mathrm{~h}$ post-infection, $\mathrm{CFU}$ from the spleen and liver were enumerated (Figure 3). WT L. monocytogenes grew to $10^{7} \mathrm{CFU}$ in both the spleen and liver, while $\Delta$ hly L. monocytogenes was extremely attenuated with bacteria from the spleen and liver near or below the limit of detection in most mice. Interestingly, $h l y{ }^{\mathrm{fl}}$ L. monocytogenes had a moderate level of attenuation. $10^{4} \mathrm{CFU}$ were recovered from the spleen, representing a statistically-significant 3-log reduction in virulence compared to WT, although a smaller reduction in virulence than that of $\Delta h l y$. In the liver, less than $10^{3} \mathrm{CFU}$ were recovered.

\subsubsection{Vaccination with $h l y$ fl Confers Protective Immunity}

The two requirements for a vaccine are safety and efficacy. In Figure 3 , we showed that $h l y^{\mathrm{fl}}$ is highly attenuated, and thus satisfies the safety requirement. To test the efficacy of $h l y^{\mathrm{fl}}$ L. monocytogenes as a vaccine, a protection study was performed. Protection of $h l y^{\mathrm{fl}}$ was compared to protection conferred by $\triangle a c t A$, which is well established as an attenuated and effective vaccine strain [21], and $\Delta h l y$, which does not confer strong protection. C57BL/6J mice were vaccinated with either $10^{3}$ or $10^{5} \mathrm{~L}$. monocytogenes. Four weeks post-vaccination, the mice were challenged with a lethal dose of WT L. monocytogenes. Three days post-challenge, CFU from the spleen were enumerated (Figure 4A). 
Vaccination with $10^{5} \mathrm{CFU}$ of $h l y^{\mathrm{fl}}$ provided 5-logs of protection, albeit less protection than $\triangle$ act $A$, while vaccination with $10^{5} \mathrm{CFU}$ of $\Delta h l y$ did not confer protection. However, vaccination with $10^{3} \mathrm{hly}$ fl was not as protective as $10^{3} \Delta a c t A$.
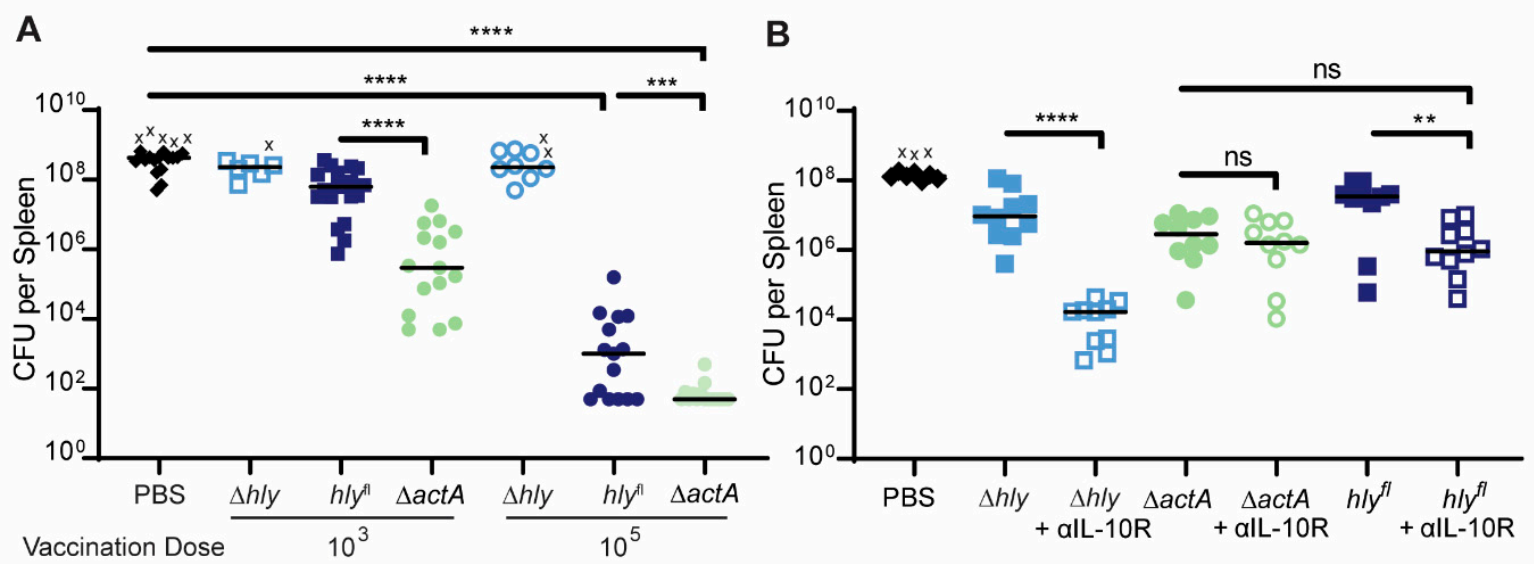

Figure 4. Vaccination with $h l y^{\mathrm{fl}}$ confers protective immunity in mice. (A) C57BL/6J mice were vaccinated intravenously with $10^{3}$ or $10^{5} \mathrm{CFU}$ of L. monocytogenes. Then, 4 weeks post-vaccination, mice were challenged intravenously with $5 \times 10^{4}-1 \times 10^{5} \mathrm{CFU}$ of WT L. monocytogenes. Three days post-challenge, CFU were enumerated from the spleen. Data are pooled from two to three independent experiments. Means were compared using Holm-Sidak's multiple comparisons test. (B) C57BL/6J mice were vaccinated intravenously with $10^{3} \Delta a c t A$ or hly $\mathrm{fl}$ L. monocytogenes or $10^{8} \Delta$ hly L. monocytogenes $+/-$ treatment with $\alpha$ IL-10R antibody. Mice were challenged intravenously with $5 \times 10^{4} \mathrm{CFU}$ of WT L. monocytogenes. Three days post-challenge, CFU were enumerated from the spleen. Data are pooled from two independent experiments. Means were compared using Holm-Sidak's multiple comparisons test. $\mathrm{X}$ indicates a mouse that died from the WT challenge before organs could be harvested.

As vaccination with vacuole-confined $\Delta h l y$ is inhibited by the secretion of the immunosuppressive cytokine IL-10 [22], we hypothesized that $h l y^{\mathrm{fl}}$ is also inhibited by IL-10 because it is defective in cell-to-cell spread and remains confined in secondary vacuoles. To test this hypothesis, we performed protection studies using an IL-10 receptor blocking antibody ( $\alpha \mathrm{IL}-10 \mathrm{R})$, which improves the protective capacity of $\Delta h l y$ [22]. Indeed, administration of $\alpha \mathrm{IL}-10 \mathrm{R}$ improved the protective capacity of $\Delta h l y$ and $h l y \mathrm{fl}$ but not $\triangle a c t A$ L. monocytogenes (Figure 4B). Furthermore, the protection conferred by $h l y^{\mathrm{fl}}$ was improved to levels similar to those conferred by vaccination with $\triangle a c t A$. Thus, hly $\mathrm{fl}^{\mathrm{fl}}$ a highly attenuated strain of L. monocytogenes capable of inducing protective immunity, though its protection is reduced compared to $\Delta a c t A$ likely due to IL-10 induction.

\section{Discussion}

As a member of the cholesterol-dependent cytolysin (CDC) family of pore-forming toxins, LLO is unique as the only CDC that is secreted by an intracellular pathogen and therefore the only CDC that primarily acts on cells from within. Therefore, LLO likely affects cells in ways that extracellular CDCs do not. Yet, because deletion of $h l y$ prevents study of the effects of LLO in the cytosol, other studies have utilized the application of exogenous purified LLO to study its effects [15-19]. Here, we described a L. monocytogenes strain that uses a Cre-lox system to delete hly following vacuolar escape. This strain, $h l y \mathrm{fl}$, became $100 \%$ non-hemolytic less than $1.5 \mathrm{~h}$ after infection of macrophages (Figure 1C) and replicated to high numbers in the cytosol (Figure 2A,E). hly excision after vacuolar escape allows the study of LLO functions in the cytosol separate from its role in vacuolar escape.

\subsection{Insights into the Effects of LLO during Infection}

LLO is a pore-forming toxin that oligomerizes and forms pores in cholesterol-containing membranes, including the host cell vacuolar and plasma membranes $[23,24]$. Secretion of LLO 
in the cytosol has the potential to damage the host cell plasma membrane. Although multiple mechanisms limit LLO damage to the cell plasma membrane, it has been difficult to establish whether these mechanisms are entirely effective in preventing cell death [7]. Here, we showed that L. monocytogenes that does not produce LLO in the cytosol replicates to greater numbers in macrophages and is less cytotoxic. The revelation that LLO secreted in the cytosol is cytotoxic makes it curious that L. monocytogenes continuously secretes LLO. It is possible that the continuous production of LLO is necessary to ensure a rapid escape from secondary vacuoles, as defects that reduce cell-to-cell spread are highly attenuated.

It is also possible that the innate immune response to LLO-induced cell death contributes to pathogenesis. In the host cell cytosol, infrequent lysis of L. monocytogenes induces pyroptotic cell death via the AIM2 inflammasome [25]. Some forms of cell death are thought to inhibit the generation of protective immunity. Strains of L. monocytogenes engineered to induce pyroptosis, necrosis or apoptosis inhibit the generation of protective immunity [26]. Thus, it is likely that LLO-induced cell death also affects the immune response to L. monocytogenes infection. The type of cell death induced by LLO and its effects on the innate and adaptive immune responses to L. monocytogenes merit further study.

Analysis of the kinetics of $h l y$ deletion, specifically the more rapid deletion in the spleen compared to the liver, revealed differences in the environments experienced by L. monocytogenes. CD169+ macrophages that are localized to the marginal zone of the spleen and dendritic cells are thought to be the first splenic cell types infected by L. monocytogenes upon intravenous inoculation $[27,28]$. Three hours post-infection, the majority of L. monocytogenes in the spleen are trapped within CD169+ macrophages [27]. Following infection with $h l y^{\mathrm{fl}}$, bacteria in the spleen became non-hemolytic at a rate similar to in BMMs, with almost complete loss of hemolytic capacity three hours post-infection, suggesting that infection of BMMs closely models infection in the spleen with respect to activation of virulence genes. However, bacteria became non-hemolytic at a much slower rate in the liver, with greater than $20 \%$ of bacteria remaining hemolytic at $5 \mathrm{~h}$ post-infection and complete loss of hemolysis between eight and $24 \mathrm{~h}$ post-infection. In the liver, four hours after infection with L. monocytogenes $100 \%$ of infected cells are tissue-resident macrophages, known as Kupffer cells [29]. Bacteria that are not killed by the Kupffer cells can transfer to hepatocytes, which become heavily infected [30]. Over the next couple days, infected Kupffer cells die by necroptosis; infiltrating neutrophils and monocyte-derived macrophages lyse infected hepatocytes and become the primarily infected cells [30-32]. It is possible that $h l y^{f l}$ became non-hemolytic slowly in the liver because act $A$ expression was not efficiently upregulated in Kupffer cells, and Cre-lox recombination only occurred after the bacteria were transferred to hepatocytes, neutrophils and/or monocyte-derived macrophages. If this is the case, it would be interesting to understand why the intracellular environment of a Kupffer cell does not activate act $A$ expression like other cells. Alternatively, the bacteria that became non-hemolytic during the first $8 \mathrm{~h}$ of infection could represent the population of bacteria inside Kupffer cells, hepatocytes and/or infiltrating neutrophils and monocyte-derived macrophages, and the bacteria that remained hemolytic may represent an extracellular population of bacteria in the liver. A small population of extracellular bacteria associated with nonparenchymal cells in the liver six hours after infection has been previously identified, though it is not clear whether these bacteria became extracellular following lysis of infected hepatocytes, or whether they never infected cells [33].

\subsection{Limitations of hly $\mathrm{fl}^{l}$}

Although we have successfully employed $h l y^{\mathrm{fl}}$ to demonstrate the contribution of LLO to cytotoxicity in macrophages, this system is limited by the fact that loss of LLO is permanent and not conditional to the environment of the cytosol. As a result, $h l y^{\mathrm{fl}}$ became trapped in secondary vacuoles and was defective in cell-to-cell spread (Figure 2C-E). Bacteria that are released from the cytosol upon cell lysis also behave like LLO-minus mutants. The inability to escape subsequent vacuoles likely explains the attenuation of $h l y^{\mathrm{fl}}$ in mice (Figure $\left.3 C, D\right)$, and why $h l y^{\mathrm{fl}}$ vaccination was improved by $\alpha \mathrm{IL}-10 \mathrm{R}$ antibody (Figure 4B). 
An additional complication of $h l y \mathrm{fl}$ is that Cre-lox recombination is susceptible to inactivation. A previous study in which a transposon library was generated in a strain of L. monocytogenes with Cre-lox identified transposon insertions in the act $A$ promoter driving cre expression and loxP sites that prevented recombination. We observed a population of hemolytic bacteria in the spleen that expanded between eight- and $24 \mathrm{~h}$ post-infection. We isolated several colonies of hemolytic bacteria $24 \mathrm{~h}$ post-infection and reinfected BMMs, and bacteria were $100 \%$ hemolytic five hours post-infection (data not shown). It is possible that these hemolytic bacteria are the progeny of a founding bacterium that had a mutation in its Cre-lox machinery that prevented recombination from occurring.

In the future, the ideal tool to study the cytosolic effects of LLO in mice would not secrete LLO in the host cell cytosol but could secrete LLO upon entry into secondary cells to continue the life cycle. Nevertheless, we believe $h l y^{\mathrm{fl}}$ is well suited for studying LLO secreted in the cytosol in cells, and insights gained from study of $h l y^{\mathrm{fl}}$ in cells can be translated to the whole animal setting using various mouse models.

\section{Conclusions}

Cre-lox recombination has been a popular tool for the study of plants and mice for many decades [34-36]. In bacteria, its use has been more limited, and it has only been used a few times in L. monocytogenes. Previously in L. monocytogenes, Cre-lox was used to generate a strain that cannot replicate following activation of the $a c t A$ promoter by flanking essential genes near the origin of replication with $\operatorname{lox} P$ sites and driving Cre expression with the act $A$ promoter [37]. This strain is highly attenuated and potently activates the CD8+ T-cell response and thus is a candidate vaccine-delivery system [38]. In another instance, a strain of L. monocytogenes that deletes act $A$ in the host cell cytosol was used to show that ActA expressed in the host cell cytosol contributes to cell-to-cell spread and simultaneously allows L. monocytogenes to avoid xenophagy [39]. This works represents the first use of Cre-lox recombination to study the function of a virulence factor that is active at temporally and spatially distinct periods. We believe that this system has the promise to uncover many effects of LLO secreted in the cytosol and could also uniquely contribute to better understanding the cellular responses to membrane damage from an intracellularly secreted pore-forming toxin.

\section{Materials and Methods}

\subsection{Construction of hly ${ }^{f l}$}

$h l y \mathrm{fl}$ was constructed by integrating two plasmids, one encoding $h l y$ and tet $L$ flanked by loxP sites and the other encoding cre downstream of the act $A$ promoter, into a $\Delta$ hly strain of L. monocytogenes. The $h l y$ and tet $L$ genes were cloned into pPL1 and flanked by lox66/lox71 loxP sites such that Cre expression resulted in excision of the region flanked by loxP sites (fl). The resulting plasmid (pPL1- $h l y^{\mathrm{fl}}$ ) was transformed into SM10 E. coli. The cre recombinase gene was previously engineered downstream of the act $A$ promoter in pPL2e, yielding the plasmid pPL2e-actA-cre, which was also transformed into SM10 E. Coli. Transconjugation was performed to integrate both plasmids into $\Delta h l y$ L. monocytogenes in a stepwise manner. First, pPL1-hly ${ }^{\mathrm{fl}}$ was transconjugated with $\Delta$ hly L. monocytogenes and transconjugate colonies that were resistant to streptomycin $(200 \mu \mathrm{g} / \mathrm{mL})$ and chloramphenicol $(7.5 \mu \mathrm{g} / \mathrm{mL})$ were selected. Second, $\Delta h l y$ pPL1-hlyfl was transconjugated with pPL2e-actA-cre, and transconjugate colonies resistant to streptomycin, chloramphenicol, and erythroymycin $(1 \mu \mathrm{g} / \mathrm{mL})$ were selected. Similarly, $\triangle a c t A h l y^{\mathrm{fl}}$ was engineered by transconjugating pPL1-hly ${ }^{\mathrm{fl}}$ and pPL2e-actA-cre into $\Delta a c t A \Delta h l y$. The $h l y^{\mathrm{fl}}$ complement strain was engineered by transconjugating $\Delta h l y$ with pPL1- $h l y^{\mathrm{fl}}$.

A control pPL1 plasmid (pPL1-tet $L^{\mathrm{fl}}$ ) encoding tet $L$, but not $h l y$, flanked by loxP sites was engineered by excising $h l y$ from pPL1-hly ${ }^{\mathrm{fl}}$. The $\Delta h l y$ control strain was engineered by transconjugating $\Delta h l y$ with pPL1-tetL. The WT control strain was engineered by transconjugating WT with pPL1-tet $L^{\mathrm{fl}}$. The $\triangle$ act $A$ control strain was engineered by transconjugating $\triangle a c t A$ with $\mathrm{pPL1}$-tet $L^{\mathrm{fl}}$. 


\subsection{Bacterial Culture}

Strains used in this study are listed in Table S1. Bacteria were grown overnight at $37{ }^{\circ} \mathrm{C}$ in Brain-Heart Infusion (BHI; BD, Sparks, MD, USA) containing $200 \mu \mathrm{g} / \mathrm{mL}$ streptomycin (GoldBio, St. Louis, MO, USA), and bacteria with tet $L$ were additionally grown in $2 \mu \mathrm{g} / \mathrm{mL}$ tetracycline (GoldBio, St. Louis, MO, USA). Overnight cultures were diluted 1:200 and grown in BHI containing streptomycin (for bacteria without tet $L$ ) or streptomycin and tetracycline (for bacteria with tet $L$ ) at $37^{\circ} \mathrm{C}$, shaking, to an optical density of 0.5 . These cultures were then pelleted by centrifugation and resuspended in phosphate-buffered solution (PBS; Gibco, Paisley, UK) containing 9\% glycerol. These cultures were then aliquoted and frozen at $-80^{\circ} \mathrm{C}$. Aliquots were thawed and used directly for experiments.

\subsection{Preparation of $M-C S F$}

The 3T3 cell media was prepared using Dulbecco's Modified Eagle Media (DMEM, Gibco, Grand Island, NY, USA) with 10\% FBS (Seradigm, US Origin), 1\% L-Glutamine (Corning, Manassas, VA, USA), and $1 \%$ Sodium pyruvate (Corning, Manassas, VA, USA), with or without $1 \times$ Penicillin Streptomycin Solution ("Pen/Strep"; Corning, Manassas, VA, USA). The 107 M-CSF-producing 3T3 cells were seeded into a T75 flask with 20mL media containing Pen/Strep and grown at $37^{\circ} \mathrm{C} 5 \% \mathrm{CO}_{2}$ (Day 1). To split cells, media was aspirated, cells were washed with warm PBS, and incubated with 0.05\% Trypsin-EDTA (Gibco, Grand Island, NY, USA) for five minutes at $37^{\circ} \mathrm{C}$. On day 4, cells were split to a T225 in $50 \mathrm{~mL}$ media containing Pen/Strep. On day 7, cells were split to five T225 flasks in media without Pen/Strep. On day 9 or 10, when cells when cells were 100\% confluent, the five T225 flasks were split into 25 T225 flasks and grown until 100\% confluent (about 3 days) and an additional two days (about 5 days total) in media without Pen/Strep. On day 14 or 15, supernatant was removed from all flasks, filter sterilized with a $0.2 \mu \mathrm{M}$ bottle filter, and stored at $4{ }^{\circ} \mathrm{C}$. Then, $50 \mathrm{~mL}$ fresh media without Pen/Strep was added back to each T225 and flasks were incubated an additional 3 days. On day 17 or 18, supernatants were collected as before, and combined with the previous supernatants. Supernatants were stored at $-20{ }^{\circ} \mathrm{C}$ and used as the source of M-CSF for bone marrow-derived macrophage preparation and culture.

\subsection{Bone Marrow-Derived Macrophage Culture}

BMM growth media was prepared using high glucose DMEM (Thermo Fisher Scientific) with 20\% Fetal Bovine Serum (Seradigm), 1\% L-glutamine (Corning), 1\% Sodium pyruvate (Corning), 14mM 2-Mercaptoethanol (Gibco; Grand Island, NY, USA), and 10\% 3T3 cell supernatant (from M-CSF-producing 3T3 cells). Macrophages were prepared from the femurs of C57BL/6J mice. Femurs were isolated, sterilized with 70\% ethanol, and crushed with a mortar and pestle in BMM growth media. Cells were strained through a $70 \mu \mathrm{M}$ filter and distributed into ten $150-\mathrm{mm}$ non-TC dishes in 30mL BMM culture medium. An additional 30mL BMM culture medium was added at day 3. After cells were incubated for a total of seven days at $37{ }^{\circ} \mathrm{C}$ with $5 \% \mathrm{CO}_{2}$, cells were harvested and frozen at $-80{ }^{\circ} \mathrm{C}$ in BMM culture medium with 10\% Fetal Bovine Serum (Seradigm) and 10\% DMSO (Sigma, St. Louis, MO, USA) added.

\subsection{Blood Agar Media}

Blood agar media was prepared using the following recipe for $1 \mathrm{~L}$ of media: $5 \mathrm{~g}$ yeast extract, $10 \mathrm{~g}$ tryptone (BTS, Richmond, CA, USA), $10 \mathrm{~g} \mathrm{NaCl}$ (Fisher, Fair Lawn, NJ, USA), $10.48 \mathrm{~g}$ MOPS (Sigma, St. Louis, MO, USA), $1 \mathrm{~g}$ activated charcoal, $5 \mathrm{~g}$ SuperPure Agar (BTS Richmond, CA, USA), $957.5 \mathrm{~mL}$ MilliQ water (Millipore Sigma, USA), and $10 \mathrm{~N} \mathrm{NaOH}$ to bring $\mathrm{pH}$ to 7.3. Media was autoclaved and cooled prior to addition of $12.5 \mathrm{~mL} 304 \mathrm{~g} / \mathrm{L}$ glucose-1-phosphate (Sigma, St. Louis, MO, USA) and 25 mL defibrinated Sheep's blood (Hemostat Laboratories, Dixon, CA, USA). 


\subsection{Hemolysis in BMMs}

A $60 \mathrm{~mm}$ non-TC dish with $1512 \mathrm{~mm}$ glass coverslips was seeded with $3 \times 10^{6}$ BMMs. The following day, the cells were infected with $2 \times 10^{5} \mathrm{CFU} \mathrm{hly} \mathrm{fl}$ for $30 \mathrm{~min}$. Then, cells were washed with PBS and BMM media with $50 \mu \mathrm{g} / \mathrm{mL}$ gentamicin was added. At each timepoint, coverslips were removed from the dish and placed in water to lyse the cells. Bacteria were plated on blood-agar media. Plates were incubated overnight at $37^{\circ} \mathrm{C}$ and then transferred to $4{ }^{\circ} \mathrm{C}$ until halos surrounding hemolytic colonies were clear. Hemolytic and ahemolytic colonies were enumerated.

\subsection{Hemolysis in Mice}

C57BL/6J (The Jackson Laboratory, Bar Harbor, ME, USA) mice were infected with $1 \times 10^{5} \mathrm{CFU}$ of $h l y^{\mathrm{fl}}$ L. monocytogenes. At 0, 1, 2, 3, 5, 8, and $24 \mathrm{~h}$ post-infection mice were euthanized (3 mice per timepoint) and spleens and livers were harvested, homogenized, and plated on blood-agar media. Plates were incubated overnight at $37^{\circ} \mathrm{C}$ and then transferred to $4{ }^{\circ} \mathrm{C}$ until halos surrounding hemolytic colonies were clear. Hemolytic and ahemolytic colonies were enumerated.

\subsection{Lactate Dehydrogenase (LDH) Assay}

BMMs were seeded into a 24-well plate with $5 \times 10^{5} \mathrm{BMMs} /$ well. The following day, cells were infected with $2 \times 10^{6} \mathrm{CFU} \mathrm{L}$. monocytogenes for $30 \mathrm{~min}$. Then, cells were washed with PBS and BMM media containing $5 \%$ FBS and $50 \mu \mathrm{g} / \mathrm{mL}$ gentamicin was added to wells. Then, $24 \mathrm{~h}$ post-infection LDH assay was performed as previously described [25].

\subsection{Intracellular Growth Curves}

In total, $3 \times 10^{6}$ BMMs were plated in $60 \mathrm{~mm}$ non-TC-treated Petri dishes with $1512 \mathrm{~mm}$ glass coverslips in each dish. The following day, two dishes per strain were infected with $5 \times 10^{5} \mathrm{CFU}$ $(\mathrm{MOI}=0.17)$ and intracellular growth curves were performed as described previously [40].

\subsection{Plaque Assay}

Six-well plates were seeded with $1.2 \times 10^{6} \mathrm{~L} 2$ cells per well. The plaque assay was performed as previously described [41].

\subsection{Cell Spreading Assay}

BMMs were seeded into a 24-well plate with glass coverslips in each well, at $3.5 \times 10^{5}$ cells/well. Three coverslips were infected for each strain. Then, $30 \mathrm{~min}$ post-infection, cells were washed with PBS and BMM media containing $50 \mu \mathrm{g} / \mathrm{mL}$ gentamicin (Sigma-Aldrich, St. Louis, MO, USA) was added. At five hours post-infection, coverslips were removed, and cells were fixed with $100 \%$ methanol and stained using Diff-Quik stain. Coverslips were mounted onto glass slides using Permount (Fisher Chemical). Light microscopy was performed using a BZ-X700 microscope (KEYENCE, Osaka, Japan) and a $60 \times$ objective lens. Cell spread index was calculated by counting the number of cells in an infectious focus containing 5 or more bacteria and subtracting one cell so that the cell spread index represents the number of cells that were spread to from the initially infected cell. Analysis was performed blindly. In total, $60 \mathrm{hlyl}$ and $67 \mathrm{WT}$ infectious foci were analyzed.

\subsection{Animal Use Ethics Statement}

All animal work was done in strict accordance with university regulations. Protocols were reviewed and approved by the Animal Care and Use Committee at the University of California, Berkeley AUP-2016-05-8811. Date of approval: 11 February 2016. 


\subsection{Virulence in Mice}

Eight-week-old female CD-1 mice (Charles River Laboratories, Wilmington, MA, USA) were infected intravenously with $1 \times 10^{5} \mathrm{CFU}$ in $200 \mu \mathrm{L}$ PBS. Forty-eight hours post-infection, the mice were euthanized, and spleens and livers were harvested, homogenized in 0.1\% IGEPAL CA-630 (Sigma, St. Louis, MO, USA) in water, and plated on LB agar (Fisher, Fair Lawn, NJ, USA) with $200 \mu \mathrm{g} / \mathrm{mL}$ streptomycin for enumeration of bacterial burdens.

\subsection{Vaccination of Mice}

Eight-to-ten-week-old female C57BL/6J mice (The Jackson Laboratory) were vaccinated by intravenous injection of L. monocytogenes in $200 \mu \mathrm{L}$ PBS. Four weeks post-vaccination, mice were challenged with $5 \times 10^{4}$ CFU WT L. monocytogenes injected intravenously in $200 \mu \mathrm{L}$ PBS. Three days post-challenge, mice were euthanized, and spleens and livers were harvested, homogenized in $0.1 \%$ IGEPAL CA-630 (Sigma), and plated on LB Agar with $200 \mu \mathrm{g} / \mathrm{mL}$ streptomycin for enumeration of bacterial burdens. Mice treated with $\alpha$ IL-10R antibody (Clone 1B1.3A, Bio X Cell, West Lebanon, NH, USA) were injected with $250 \mu \mathrm{g}$ of antibody in $100 \mu \mathrm{L}$ PBS two hours prior to vaccination.

\subsection{Statistical Analysis}

Data were analyzed using GraphPad Prism 8 (GraphPad, San Diego, CA, USA, 2019). For mouse CFU experiments, data were log transformed prior to performing statistical analysis. ${ }^{*}$ indicates $p<0.05 ;{ }^{* *}$ indicates $p<0.01 ;{ }^{* * *}$ indicates $p<0.001 ;{ }^{* * *}$ indicates $p<0.0001$; ns indicates no statistical significance.

Supplementary Materials: The following are available online at http://www.mdpi.com/2072-6651/12/1/38/s1, Table S1: Strains.

Author Contributions: Conceptualization, B.N.N. and D.A.P.; Data curation, B.N.N.; Formal analysis, B.N.N.; Funding acquisition, B.N.N. and D.A.P.; Investigation, B.N.N.; Methodology, B.N.N. and D.A.P.; Project administration, B.N.N. and D.A.P.; Resources, B.N.N.; Supervision, D.A.P.; Validation, B.N.N.; Visualization, B.N.N.; Writing—original draft, B.N.N.; Writing—review \& editing, B.N.N. and D.A.P. All authors have read and agreed to the published version of the manuscript.

Funding: This work was supported by National Institutes of Health grants 1P01 AI063302, and 1R01 AI027655, and National Science Foundation, Graduate Research Fellowship DGE 1106400.

Acknowledgments: We would like to thank Chen Chen, Eric Lee and Aaron Whiteley for helpful discussion and review of this manuscript.

Conflicts of Interest: The authors declare no conflict of interest.

\section{References}

1. Falkow, S. Molecular Koch's postulates applied to microbial pathogenicity. Rev. Infect. Dis. 1988, 10, S274-S276. [PubMed]

2. Jones, S.; Portnoy, D.A. Characterization of Listeria monocytogenes pathogenesis in a strain expressing perfringolysin $\mathrm{O}$ in place of listeriolysin O. Infect. Immun. 1994, 62, 5608-5613. [CrossRef] [PubMed]

3. Moors, M.A.; Levitt, B.; Youngman, P.; Portnoy, D.A. Expression of listeriolysin O and ActA by intracellular and extracellular Listeria monocytogenes. Infect. Immun. 1999, 67, 131-139. [CrossRef] [PubMed]

4. Gedde, M.M.; Higgins, D.E.; Tilney, L.G.; Portnoy, D.A. Role of listeriolysin O in cell-to-cell spread of Listeria monocytogenes. Infect. Immun. 2000, 68, 999-1003. [CrossRef] [PubMed]

5. Dancz, C.E.; Haraga, A.; Portnoy, D.A.; Higgins, D.E. Inducible control of virulence gene expression in Listeria monocytogenes: Temporal requirement of listeriolysin O during intracellular infection. J. Bacteriol. 2002, 184, 5935-5945. [CrossRef] [PubMed]

6. Los, F.C.O.; Randis, T.M.; Aroian, R.V.; Ratner, A.J. Role of pore-forming toxins in bacterial infectious diseases. Microbiol. Mol. Biol. Rev. 2013, 77, 173-207. [CrossRef] [PubMed]

7. Nguyen, B.N.; Peterson, B.N.; Portnoy, D.A. Listeriolysin O: A phagosome-specific cytolysin revisited. Cell. Microbiol. 2019, 21, e12988. [CrossRef] 
8. Glomski, I.J.; Gedde, M.M.; Tsang, A.W.; Swanson, J.A.; Portnoy, D.A. The Listeria monocytogenes hemolysin has an acidic $\mathrm{pH}$ optimum to compartmentalize activity and prevent damage to infected host cells. J. Cell Biol. 2002, 156, 1029-1038. [CrossRef]

9. Schnupf, P.; Portnoy, D.A.; Decatur, A.L. Phosphorylation, ubiquitination and degradation of listeriolysin O in mammalian cells: Role of the PEST-like sequence. Cell. Microbiol. 2006, 8, 353-364. [CrossRef]

10. Schnupf, P.; Portnoy, D.A. Listeriolysin O: A phagosome-specific lysin. Microbes Infect. 2007, 9, $1176-1187$. [CrossRef]

11. Schnupf, P.; Hofmann, J.; Norseen, J.; Glomski, I.J.; Schwartzstein, H.; Decatur, A.L. Regulated translation of listeriolysin O controls virulence of Listeria monocytogenes. Mol. Microbiol. 2006, 61, 999-1012. [CrossRef] [PubMed]

12. Chen, C.; Nguyen, B.N.; Mitchell, G.; Margolis, S.R.; Ma, D.; Portnoy, D.A. The Listeriolysin O PEST-like Sequence Co-opts AP-2-Mediated Endocytosis to Prevent Plasma Membrane Damage during Listeria Infection. Cell Host Microbe 2018, 23, 786-795. [CrossRef] [PubMed]

13. Glomski, I.J.; Decatur, A.L.; Portnoy, D.A. Listeria monocytogenes Mutants That Fail to Compartmentalize Listerolysin O Activity Are Cytotoxic, Avirulent, and Unable to Evade Host Extracellular Defenses. Infect. Immun. 2003, 71, 6754-6765. [CrossRef] [PubMed]

14. Czuczman, M.A.; Fattouh, R.; Van Rijn, J.M.; Canadien, V.; Osborne, S.; Muise, A.M.; Kuchroo, V.K.; Higgins, D.E.; Brumell, J.H. Listeria monocytogenes exploits efferocytosis to promote cell-to-cell spread. Nature 2014, 509, 230-234. [CrossRef] [PubMed]

15. Hamon, M.A.; Ribet, D.; Stavru, F.; Cossart, P. Listeriolysin O: The Swiss army knife of Listeria. Trends Microbiol. 2012, 20, 360-368. [CrossRef]

16. Li, J.; Lam, W.W.; Lai, T.; Au, S.W. Degradation of nuclear Ubc9 induced by listeriolysin O is dependent on K + efflux. Biochem. Biophys. Res. Commun. 2017, 493, 1115-1121. [CrossRef]

17. Stavru, F.; Bouillaud, F.; Sartori, A.; Ricquier, D.; Cossart, P. Listeria monocytogenes transiently alters mitochondrial dynamics during infection. Proc. Natl. Acad. Sci. USA 2011, 108, 3612-3617. [CrossRef]

18. Hamon, M.A.; Batsché, E.; Régnault, B.; Tham, T.N.; Seveau, S.; Muchardt, C.; Cossart, P. Histone modifications induced by a family of bacterial toxins. Proc. Natl. Acad. Sci. USA 2007, 104, 13467-13472. [CrossRef]

19. Osborne, S.E.; Brumell, J.H. Listeriolysin O: From bazooka to Swiss army knife. Philos. Trans. R. Soc. Lond. B. Biol. Sci. 2017, 372, 20160222. [CrossRef]

20. Brundage, R.A.; Smith, G.A.; Camilli, A.; Theriot, J.A.; Portnoy, D.A. Expression and phosphorylation of the Listeria monocytogenes actA protein in mammalian cells. Proc. Natl. Acad. Sci. USA 1993, 90, 11890-11894. [CrossRef]

21. Goossens, P.L.; Milon, G.; Bevan, M. Induction of protective CD8 ${ }^{+} \mathrm{T}$ lymphocytes by an attenuated listeria monocytogenes actA mutant. Int. Immunol. 1992, 4, 1413-1418. [CrossRef] [PubMed]

22. Bahjat, K.S.; Meyer-Morse, N.; Lemmens, E.E.; Shugart, J.A.; Dubensky, T.W.; Brockstedt, D.G.; Portnoy, D.A. Suppression of cell-mediated immunity following recognition of phagosome-confined bacteria. PLoS Pathog. 2009, 5, e1000568. [CrossRef] [PubMed]

23. Gilbert, R.J.C. Cholesterol-Dependent Cytolysins. In Proteins Membrane Binding and Pore Formation; Springer: New York, NY, USA, 2010. Available online: https://link.springer.com/chapter/10.1007\%2F978-1-4419-63277_5 (accessed on 21 January 2020).

24. Mulvihill, E.; Van Pee, K.; Mari, S.A.; Muller, D.J.; Yildiz, O. Directly Observing the Lipid-Dependent Self-Assembly and Pore-Forming Mechanism of the Cytolytic Toxin Listeriolysin O. Nano Lett. 2015, 15, 6965-6973. [CrossRef] [PubMed]

25. Sauer, J.D.; Witte, C.E.; Zemansky, J.; Hanson, B.; Lauer, P.; Portnoy, D.A. Listeria monocytogenes triggers AIM2-mediated pyroptosis upon infrequent bacteriolysis in the macrophage cytosol. Cell Host Microbe 2010, 7, 412-419. [CrossRef]

26. Theisen, E.; Sauer, J.-D. Listeria monocytogenes-Induced Cell Death Inhibits the Generation of Cell-Mediated Immunity. Infect. Immun. 2017, 85, e00733-16. [CrossRef]

27. Perez, O.A.; Yeung, S.T.; Vera-Licona, P.; Romagnoli, P.A.; Samji, T.; Ural, B.B.; Maher, L.; Tanaka, M.; Khanna, K.M. CD169+ macrophages orchestrate innate immune responses by regulating bacterial localization in the spleen. Sci. Immunol. 2017, 2, eaah5520. [CrossRef] 
28. Waite, J.C.; Leiner, I.; Lauer, P.; Rae, C.S.; Barbet, G.; Zheng, H.; Portnoy, D.A.; Pamer, E.G.; Dustin, M.L. Dynamic imaging of the effector immune response to listeria infection In Vivo. PLoS Pathog. 2011, 7, e1001326. [CrossRef]

29. Blériot, C.; Dupuis, T.; Jouvion, G.; Eberl, G.; Disson, O.; Lecuit, M. Liver-Resident Macrophage Necroptosis Orchestrates Type 1 Microbicidal Inflammation and Type-2-Mediated Tissue Repair during Bacterial Infection. Immunity 2015, 42, 145-158. [CrossRef]

30. Conlan, J.W.; North, R.J. Early pathogenesis of infection in the liver with the facultative intracellular bacteria Listeria monocytogenes, Francisella tularensis, and Salmonella typhimurium involves lysis of infected hepatocytes by leukocytes. Infect. Immun. 1992, 60, 5164-5171. [CrossRef]

31. North, R.J. The relative importance of blood monocytes and fixed macrophages to the expression of cell-mediated immunity to infection. J. Exp. Med. 1970, 132, 521-534. [CrossRef]

32. Lepay, D.A.; Steinman, R.M.; Nathan, C.F.; Murray, H.W.; Cohn, Z.A. Liver macrophages in murine listeriosis: Cell-mediated immunity is correlated with an influx of macrophages capable of generating reactive oxygen intermediates. J. Exp. Med. 1985, 161, 1503-1512. [CrossRef] [PubMed]

33. Gregory, S.H.; Sagnimeni, A.J.; Wing, E.J. Bacteria in the bloodstream are trapped in the liver and killed by immigrating neutrophils. J. Immunol. 1996, 157, 2514-2520. [PubMed]

34. Gilbertson, L. Cre-lox recombination: Cre-ative tools for plant biotechnology. Trends Biotechnol. 2003, 21, 550-555. [CrossRef] [PubMed]

35. Mortensen, R. Overview of Gene Targeting by Homologous Recombination. In Current Protocols in Molecular Biology; John Wiley \& Sons, Inc.: Hoboken, NJ, USA, 2006. Available online: https:/currentprotocols. onlinelibrary.wiley.com/doi/abs/10.1002/0471142727.mb2301s76 (accessed on 21 January 2020).

36. Yarmolinsky, M.; Hoess, R. The Legacy of Nat Sternberg: The Genesis of Cre-lox Technology. Annu. Rev. Virol. 2015, 2, 25-40. [CrossRef]

37. Reniere, M.L.; Whiteley, A.T.; Hamilton, K.L.; John, S.M.; Lauer, P.; Brennan, R.G.; Portnoy, D.A. Glutathione activates virulence gene expression of an intracellular pathogen. Nature 2015, 517, 170-173. [CrossRef]

38. Hanson, W.G.; Benanti, E.L.; Lemmens, E.E.; Liu, W.; Skoble, J.; Leong, M.L.; Rae, C.S.; Fassò, M.; Brockstedt, D.G.; Chen, C.; et al. A Potent and Effective Suicidal Listeria Vaccine Platform. Infect. Immun. 2019, 87, e00144-19. [CrossRef]

39. Mitchell, G.; Cheng, M.I.; Chen, C.; Nguyen, B.N.; Whiteley, A.T.; Kianian, S.; Cox, J.S.; Green, D.R.; McDonald, K.L.; Portnoy, D.A. Listeria monocytogenes triggers noncanonical autophagy upon phagocytosis, but avoids subsequent growth-restricting xenophagy. Proc. Natl. Acad. Sci. USA 2017, 115, E210-E217. [CrossRef]

40. Portnoy, D.A.; Jacks, P.S.; Hinrichs, D.J. Role of hemolysin for the intracellular growth of Listeria monocytogenes. J. Exp. Med. 1988, 167, 1459-1471. [CrossRef]

41. Sun, A.N.; Camilli, A.; Portnoy, D.A. Isolation of Listeria monocytogenes small-plaque mutants defective for intracellular growth and cell-to-cell spread. Infect. Immun. 1990, 5, 3770-3778. [CrossRef]

(C) 2020 by the authors. Licensee MDPI, Basel, Switzerland. This article is an open access article distributed under the terms and conditions of the Creative Commons Attribution (CC BY) license (http://creativecommons.org/licenses/by/4.0/). 